\title{
RANGE TRANSFORMATIONS ON A BANACH FUNCTION ALGEBRA. IV
}

\author{
OSAMU HATORI
}

(Communicated by Palle E. T. Jorgensen)

\begin{abstract}
Functions in $\operatorname{Op}\left(I_{D}, \operatorname{Re} A+L\right)$ are harmonic on $D$ for a closed subalgebra $A$ of $C_{0}(Y)$, an ideal $I$ of $A$ and a linear subspace $L$ of finite dimension in $C_{0, R}(Y)$ unless the uniform closure of $I$ is selfadjoint.
\end{abstract}

\section{INTRODUCTION}

Let $Y$ be a locally compact Hausdorff space, and $A$ be a uniformly closed subalgebra of $C_{0}(Y)$. We have shown in [5, Theorem 2] that functions in $\mathrm{Op}\left(I_{D}, \operatorname{Re} A\right)$ for certain ideals $I$ of a closed subalgebra $A$ of $C_{0}(Y)$, and a plane domain $D$ containing the origin, are harmonic near the origin. In this paper we show that the functions are harmonic on the whole of $D$ for more general range transformations. The proof of Theorem 2 in [5] depends on analysis of the behavior of functions near each point of $Y-\operatorname{Ker} I$. In this paper we show that it is enough to analyze near the strong boundary points.

Notations and terminologies are essentially due to [5], while in this paper, $\|\cdot\|_{\infty(K)}$ denotes the uniform norm on a set $K$, which is simply denoted by $\|\cdot\|_{\infty}$ in [5].

Lemma 1. Let $X$ be a compact Hausdorff space, $A$ a closed subalgebra of $\{f \in$ $C(X): f \mid K=0\}$ for a (possibly empty) compact subset $K$ of $X$ which satisfies that for any pair of different points $x$ in $X-K$ and $y$ in $X$, there exists $f$ in $A$ with $f(x) \neq f(y)$. Suppose that each $x$ in the Choquet boundary $\operatorname{Ch}(A)$ for $A$, with at most finitely many exceptions, has a compact neighborhood $G_{x}$ such that $A \mid G_{x}=C\left(G_{x}\right)$. Then $A$ coincides with $\{f \in C(X): f \mid K=0\}$.

Proof. We may assume $K \neq X$. If $K$ is empty, then we add $\infty$ as an isolated point and $X^{\prime}$ denotes $X \cup\{\infty\}$ and $A^{\prime}$ denotes $\left\{f \in C\left(X^{\prime}\right): f(\infty)=0, f \mid X \in\right.$ $A\}$. If $K$ is not empty, then $X^{\prime}$ denotes the quotient space of $X$ obtained by identifying points in $K$, and we denote the point in $X^{\prime}$ which corresponds to $K$ by $\infty$. We may suppose that $A$ is a closed subalgebra of $\left\{f \in C\left(X^{\prime}\right): f(\infty)=\right.$ $0\}$. We write $A^{\prime}$ instead of $A$ if we view $A$ as an algebra on $X^{\prime}$. In any case $A^{\prime}$ is a point separating uniformly closed subalgebra of $\left\{f \in C\left(X^{\prime}\right): f(\infty)=0\right\}$.

Received by the editors January 30, 1991 and, in revised form, February 19, 1991.

1980 Mathematics Subject Classification (1985 Revision). Primary 46J10.

Key words and phrases. Function algebra, range transformation, ultraseparating. 
Let $B$ denote the function algebra $\left\{f \in C\left(X^{\prime}\right): f=g+c\right.$ for $g \in A^{\prime}$ and a constant function $c$ on $\left.X^{\prime}\right\}$. If $B$ coincides with $C\left(X^{\prime}\right)$, then we see that $A^{\prime}=\left\{f \in C\left(X^{\prime}\right): f(\infty)=0\right\}$, in particular, $A=\{f \in C(X): f \mid K=0\}$. Thus we will show that $B=C\left(X^{\prime}\right)$. First we show that the family of peak sets for $A^{\prime}$ coincides with the family of peak sets for $B$ which do not contain $\infty$. Every peak set for $A^{\prime}$ is trivially a peak set for $B$ without $\infty$, so we prove the inverse implication. Let $P$ be a peak set for $B$ with $\infty$ off $P$ and $g$ a peaking function for $P$, that is, $g$ is a function in $B$ with $\|g\|_{\infty\left(X^{\prime}\right)}=1$ such that $g=1$ on $P$ and $|g|<1$ off $P$. Without loss of generality we may suppose that $0 \leq|g(\infty)|<\frac{1}{3}$. Put $h=(g-g(\infty)) /(1-g(\infty))$. We see that $h$ is a function in $A^{\prime}$ with $h=1$ on $P$ and $\|h\|_{\infty} \leq 2$. For every nonnegative integer $n$ put $G_{n}=\left\{x \in X^{\prime}:|h(x)| \leq 1+2^{-n}\right\}$. Then $G_{n}$ is a compact neighborhood of $P$ with $G_{n} \supset G_{n+1}$ for every $n$. Since each Int $G_{n}$ includes $P$, we have

$$
\sup \left\{|g(x)|: x \in X^{\prime}-G_{n}\right\}=\delta_{n}<1
$$

for every positive integer $n$. Choose positive integers $m(n)$ with $\delta_{n}^{m(n)}<$ $2^{-(n+1)}$. We see that $f_{N}=\sum_{n=1}^{N} 2^{-n} h g^{m(n)}$ is in $A^{\prime}$ for every positive integer $N$ since $B \cdot A^{\prime} \subset A^{\prime}$ and that $f_{N}$ converges to a peaking function $f$ for $P$ in $A^{\prime}$. We conclude that the two families of peak sets coincide. It follows that $\operatorname{Ch}(B)-\{\infty\}=\operatorname{Ch}\left(A^{\prime}\right)$. We may suppose that $\operatorname{Ch}(A)=\operatorname{Ch}\left(A^{\prime}\right)$. Let $\left\{X_{\alpha}\right\}$ be the maximal antisymmetric decomposition of $X^{\prime}$ for $B$. We see that $\mathrm{Ch}\left(B \mid X_{\alpha}\right)=\operatorname{Ch}(B) \cap X_{\alpha}$ for every $X_{\alpha}$ since $X_{\alpha}$ is an intersection of peak sets. We show that every $X_{\alpha}$ is singleton. Suppose not. Choose $X_{\alpha}$ which is not singleton. Then $X_{\alpha}$ contains an infinite number of points in $\operatorname{Ch}\left(B \mid X_{\alpha}\right)$. By the condition on $\operatorname{Ch}(A)$ there is a point $x$ in $\operatorname{Ch}\left(B \mid X_{\alpha}\right)$ which has a compact neighborhood $G_{x}$ in $X^{\prime}$ with $B\left|G_{x} \supset A\right| G_{x}=C\left(G_{x}\right)$. Thus $X_{\alpha} \cap G_{x}$ is an interpolating compact neighborhood of $x$ for $B \mid X_{\alpha}$. It follows by Lemma 2.5 in [2] that $X_{\alpha}$ is a single point, which is a contradiction. Thus we see that every $X_{\alpha}$ is a finite set, so every $X_{\alpha}$ is a one point set. We conclude that $B=C\left(X^{\prime}\right)$ by Bishop's theorem.

Lemma 2. Let $F$ be a compact Hausdorff space and $Q$ a compact subset (possibly empty) of $F$. Let $S$ be a Banach algebra contained in $\{f \in C(F): f \mid Q=0\}$ with the norm $\|\cdot\|_{S}$ such that $S$ separates a point $x$ in $F$ and a point $y$ in $F-Q$ if $x \neq y$. Suppose that $Q=\{x \in F: f(x)=0$ for every $f$ in $S\}$. Suppose that $\widetilde{S}^{\Lambda}$ (with respect to $\|\cdot\|_{S}$ ) separates the different points in $F_{x}^{\Lambda}$ for every $x \in F-Q$ and a discrete space $\Lambda$ with cardinality not less than that of an open base for the topology of $Y$ at $x$. Let $\varepsilon$ be a positive number and $h_{0} a$ continuous real valued function on $\{z \in \mathbf{C}:|z| \leq \varepsilon\}$ which is not harmonic on any open neighborhood of the origin. Let $T$ be a Banach space with the norm $\|\cdot\|_{T}$ continuously embedded in $C_{R}(F)$, that is, the identity map from $T$ into $C_{R}(F)$ is continuous. Let $S_{0}$ be a dense (with respect to the topology induced by $\|\cdot\|_{S}$ ) subset of $\left\{f \in S:\|f\|_{S}<\delta\right\}$ for a positive number $\delta$ with $\delta<\varepsilon$. Suppose that the function $h_{0}(f)$ is in $T$ for every $f \in S_{0}$. Then for every point $x$ in $F-Q$ there exists a compact neighborhood $G$ of $x$ with $G \subset F-Q$ which satisfies $\{f \in T: f \mid Q=0\} \mid G=C_{R}(G)$.

Proof. We denote $T_{0}=\{f \in T: f \mid Q=0\}$ and $T_{1}=\{f \in T: f \mid Q$ is constant $\}$ with the norm $\|\cdot\|_{T}$ restricted to $T_{0}$ and $T_{1}$ respectively. We will 
show that $\operatorname{cl}\left(\widetilde{T}_{0}^{\Lambda} \mid F_{x}^{\Lambda}\right)=C_{R}\left(F_{x}^{\Lambda}\right)$ for a point $x$ in $F-Q$ and a discrete space $\Lambda$ with cardinality not less than that of an open base for the topology of $Y$ at $x$. It follows that $T_{0} \mid G=C_{R}(G)$ for a compact neighborhood $G$ of $x$ with $G \subset F-Q$ by (2) of Theorem 1 in [5]. (The theorem is stated for a Banach space included (see [5, p. 89] for the definition) in $C_{0}(Y)$ or $C_{0, R}(Y)$ in [5]. Every Banach space continuously embedded in $C_{0}(Y)$ or $C_{0, R}(Y)$ has a norm equivalent to that of a Banach space included in $C_{0}(Y)$ or $C_{0, R}(Y)$. So the theorem is also true for Banach spaces continuouly embedded in $C_{0}(Y)$ or $C_{0, R}(Y)$.) Take a point $x$ in $F-Q$. Then there is $f$ in $S$ with $f(x)=1$. For some $\eta$ with $0<\eta<\varepsilon /\left(2\|f\|_{S}\right)$, and some smoothing operator $\sigma_{\eta}(w)$ of class $C^{\infty}$ supported in $\{z \in \mathbf{C}:|z|<\eta\}$, we have $\Delta_{1}\left(h_{\eta}(0,1)\right) \neq 0$ (see $[3, \mathrm{p}$. 566; 4, pp. 634, 635; 5, p. 115], where

$$
h_{\eta}\left(z_{1}, z_{2}\right)=\iint h_{0}\left(z_{1}-z_{2} w\right) \sigma_{\eta}(w) d x d y .
$$

Choose a discrete space $\Lambda$ with cardinality not less than that of an open base for the topology of $Y$ at $x$. Let $\tilde{g}$ be a function in $\widetilde{S}^{\Lambda}$. For a complex number $\beta$ with sufficiently small absolute value and a complex number $w$ with $|w|<\eta$, $h_{0}\left(\tilde{g}\langle f\rangle^{2} \beta-\langle f\rangle w\right)$ is in $\mathrm{cl} \widetilde{T}_{1}^{\Lambda}$ since $S_{0}$ is dense (with respect to the topology induced by $\left.\|\cdot\|_{S}\right)$ in $\left\{f \in S:\|f\|_{S} \leq \delta\right\}$ and $\|\cdot\|_{\infty\left(\widetilde{F}^{\Lambda}\right)} \leq\|\cdot\|_{\widetilde{S}^{\Lambda}}$. Thus we have by Lemma 5 in [4], that $|\tilde{g}|^{2} \Delta_{1}\left(h_{\eta}(0,\langle f\rangle) \cdot|\langle f\rangle|^{4}\right) \in\left\{\tilde{\psi} \in \operatorname{cl} \widetilde{T}_{1}^{\Lambda}: \tilde{\psi} \mid \widetilde{Q}^{\Lambda}=0\right\}$ since $\tilde{g}=0$ on $\widetilde{Q}^{\Lambda}$. We show that $\operatorname{cl} \widetilde{T}_{0}^{\Lambda}=\left\{\tilde{\psi} \in \operatorname{cl} \widetilde{T}_{1}^{\Lambda}: \tilde{\psi} \mid \widetilde{Q}^{\Lambda}=0\right\}$. When $T_{1}=T_{0}$ it is trivial. Suppose that $T_{1}-T_{0} \neq \phi$. Let $\tilde{\psi}=\left\langle\psi_{\lambda}\right\rangle$ be in cl $\widetilde{T}_{1}^{\Lambda}$ with $\tilde{\psi} \mid \tilde{Q}^{\Lambda}=0$. For every $\xi>0$ there is $\left\langle\psi_{\lambda}^{(\xi)}\right\rangle$ in $\tilde{T}_{1}^{\Lambda}$ such that

$$
\left\|\left\langle\psi_{\lambda}\right\rangle-\left\langle\psi_{\lambda}^{(\xi)}\right\rangle\right\|_{\infty\left(\widetilde{F}^{\wedge}\right)}<\xi \text {. }
$$

Let $\psi$ be a function in $T_{1}-T_{0}$. We may assume that $\psi \mid Q=1$. So

$$
\left\langle\psi_{\lambda}^{(\xi)}-\psi_{\lambda}^{(\xi)}(Q) \psi\right\rangle \in \widetilde{T}_{0}^{\Lambda}
$$

and

$$
\left\|\left\langle\psi_{\lambda}\right\rangle-\left(\left\langle\psi_{\lambda}^{(\xi)}-\psi_{\lambda}^{(\xi)}(Q) \psi\right\rangle\right)\right\|_{\infty\left(\widetilde{F}^{\Lambda}\right)}<\xi\left(1+\|\psi\|_{\infty(F)}\right),
$$

since $\psi_{\lambda}^{(\xi)}(Q)$ is a constant with $\left|\psi_{\lambda}^{(\xi)}(Q)\right| \leq\left\|\left\langle\psi_{\lambda}\right\rangle-\left\langle\psi_{\lambda}^{(\xi)}\right\rangle\right\|_{\infty\left(\widetilde{F}^{\Lambda}\right)^{\text {. }}}$. Thus the conclusion follows. We see that $|\tilde{g}|^{2} \Delta_{1}\left(h_{\eta}(0,\langle f\rangle) \cdot|\langle f\rangle|^{4}\right)$ is in cl $\widetilde{T}_{0}^{\Lambda}$, so we have $|\tilde{g}|^{2} \Delta_{1}\left(h_{\eta}(0,1)\right)\left|F_{x}^{\Lambda} \in\left(\operatorname{cl} \widetilde{T}_{0}^{\Lambda}\right)\right| F_{x}^{\Lambda} \subset \operatorname{cl}\left(\widetilde{T}_{0}^{\Lambda} \mid F_{x}^{\Lambda}\right)$, since $\langle f\rangle=1$ on $F_{x}^{\Lambda}$ by Lemma 4 in [5]. So $|\tilde{g}|^{2} \mid F_{x}^{\Lambda} \in \operatorname{cl}\left(\widetilde{T}_{0}^{\Lambda} \mid F_{x}^{\Lambda}\right)$ since $\Delta_{1}\left(h_{\eta}(0,1)\right) \neq 0$. It follows that the algebra generated by $|\tilde{g}|^{2} \mid F_{x}^{\Lambda}$ for $\tilde{g} \in \widetilde{S}^{\Lambda}$ is contained in $\operatorname{cl}\left(\widetilde{T}_{0}^{\Lambda} \mid F_{x}^{\Lambda}\right)$ since $\widetilde{S}^{\Lambda}$ is an algebra. Let $p$ and $q$ be different points in $F_{x}^{\Lambda}$. There is $\tilde{u}$ in $\widetilde{S}^{\Lambda}$ with $\tilde{u}(p) \neq \tilde{u}(q)$ and there is $v$ in $S$ with $v(x)=\tilde{u}(p)$. So $\tilde{u}-\langle v\rangle$ is in $\tilde{S}^{\Lambda}$ and $|\tilde{u}-\langle v\rangle|^{2}$ separates $p$ and $q$ since $\langle v\rangle(p)=\langle v\rangle(q)=v(x)$ by Lemma 4 in [5]. It follows by the Stone-Weierstrass theorem that $\operatorname{cl}\left(\widetilde{T}_{0}^{\Lambda} \mid F_{x}^{\Lambda}\right)=C_{R}\left(F_{x}^{\Lambda}\right)$.

Lemma 3. Let $E$ be a complex (resp. real) Banach space continuously embedded in $C(X)$ (resp. $C_{R}(X)$ ), and let $L$ be a finite-dimensional complex (resp. real) subspace of $C(X)$ (resp. $C_{R}(X)$ ) such that $E \cap L=\{0\}$. Let $\Lambda$ be a discrete space. Then $E+L$ is a Banach space with respect to the norm defined by

$$
\|u+v\|_{E+L}=\|u\|_{E}+\|v\|_{\infty},
$$


where $\|\cdot\|_{E}$ is the norm on $E$. Suppose that $x$ is a point in $X$ with $E_{x} \neq E$. Then

$$
(E+L)^{\sim \Lambda}\left|F_{x}^{\Lambda}=\widetilde{E}^{\Lambda}\right| F_{x}^{\Lambda} .
$$

Proof. It is trivial that $\|\cdot\|_{E+L}$ is a complete norm since $L$ is finite dimensional. $(E+L)^{\sim \Lambda} \supset \widetilde{E}^{\Lambda}$ is also trivial, so $(E+L)^{\sim \Lambda}\left|F_{x}^{\Lambda} \supset \widetilde{E}^{\Lambda}\right| F_{x}^{\Lambda}$. We prove the inverse inclusion. Suppose that $\left\{k_{1}, \ldots, k_{n}\right\}$ is a base for $L$. Let $\left\langle u_{\lambda}+v_{\lambda}\right\rangle \in(E+L)^{\sim \Lambda}$, where $u_{\lambda} \in E$ and $v_{\lambda}=\sum_{i=1}^{n} a_{i}^{(\lambda)} k_{i}$. Then $\left\langle u_{\lambda}\right\rangle \in \widetilde{E}^{\Lambda}$ and $\sup \left\|v_{\lambda}\right\|_{\infty(X)}<\infty$ by the definition of $\|\cdot\|_{E+L}$. Let $u$ be a function in $E$ with $u(x)=1$. Since the linear functional defined by $\sum_{i=1}^{n} a_{i} k_{i} \rightarrow a_{j}$, is continuous for every $j$, we see that $\left\langle u_{\lambda}\right\rangle+\sum_{i=1}^{n}\left\langle a_{i}^{(\lambda)} k_{i}(x) u\right\rangle$ is in $\widetilde{E}^{\Lambda}$. We see that

$$
\begin{gathered}
\left\langle u_{\lambda}\right\rangle\left|F_{x}^{\Lambda}+\sum_{i=1}^{n}\left\langle a_{i}^{(\lambda)} k_{i}(x) u\right\rangle\right| F_{x}^{\Lambda}=\left\langle u_{\lambda}\right\rangle\left|F_{x}^{\Lambda}+\sum_{i=1}^{n}\left\langle a_{i}^{(\lambda)} k_{i}\right\rangle\right| F_{x}^{\Lambda} \\
=\left\langle u_{\lambda}\right\rangle\left|F_{x}^{\Lambda}+\left\langle\sum_{i=1}^{n} a_{i}^{(\lambda)} k_{i}\right\rangle\right| F_{x}^{\Lambda}=\left\langle u_{\lambda}+v_{\lambda}\right\rangle \mid F_{x}^{\Lambda}
\end{gathered}
$$

by Lemma 4 of [5].

Lemma 4. Let $E$ be an ultraseparating complex (resp. real) Banach space continuously embedded in $C(X)$ (resp. $C_{R}(X)$ ) for a compact Hausdorff space $X$, i.e., $\|f\|_{\infty} \leq M\|f\|_{E}$ for every $f$ in $E$. Let $x$ be a point in $X$. Let $\Lambda$ and $\Lambda^{\prime}$ be infinite discrete spaces. If $p \in F_{x}^{\Lambda}$ and $q \in F_{x}^{\Lambda}-[\{x\} \times \Lambda]$ are different points, then $\widetilde{E}_{x}^{\Lambda}$ separates $p$ and $q$. If $q \in F_{x}^{\Lambda}-[\{x\} \times \Lambda]$, then $\left(\widetilde{E}_{x}^{\Lambda}\right)^{\sim \Lambda^{\prime}}$ separates the different points in $F_{q}^{\Lambda^{\prime}}$.

Proof. If $p \in[\{x\} \times \Lambda]$ and $q \in F_{x}^{\Lambda}-[\{x\} \times \Lambda]$, then there is $\tilde{g} \in \widetilde{E}_{x}^{\Lambda}$ such that $\tilde{g}(q) \neq \tilde{g}(p)=0$ by Proposition 2 in [5]. Suppose that $p$ and $q$ are different points in $F_{x}^{\Lambda}-[\{x\} \times \Lambda]$. Since $E$ is ultraseparating, there is $\tilde{f}=\left\langle f_{\lambda}\right\rangle$ in $\widetilde{E}^{\Lambda}$ with $\tilde{f}(p) \neq \tilde{f}(q)$. Put $h_{\lambda}=f_{\lambda}-f_{\lambda}(x) \cdot u$, where $u \in E$ with $u(x)=1$. (Such a function $u$ exists since $\widetilde{E}^{\Lambda}$ separates $(x, \lambda)$ and $\left(x, \lambda^{\prime}\right)$ for different $\lambda$ and $\lambda^{\prime}$.) So $\left\langle h_{\lambda}\right\rangle \in \widetilde{E}_{x}^{\Lambda}$. Take $\tilde{g} \in \widetilde{E}_{x}^{\Lambda}$ with $\tilde{g}(p) \neq 0$. Then $\left\langle h_{\lambda}\right\rangle$ or $\tilde{g}$ or $\left\langle f_{\lambda}(x)\right\rangle \cdot \tilde{g}$ separates $p$ and $q$. Let $q$ be a point in $F_{x}^{\Lambda}-[\{x\} \times \Lambda]$. By Corollary 1 of [5], $\left(\widetilde{E}^{\Lambda}\right)^{\sim \Lambda^{\prime}}$ separates the different points of $\left(\widetilde{X}^{\Lambda}\right)^{\sim \Lambda^{\prime}}$, in particular, if $\alpha$ and $\beta$ are different points in $F_{q}^{\Lambda^{\prime}}$, then there is $\tilde{\tilde{f}}=\left\langle\left\langle f_{\lambda, \lambda^{\prime}}\right\rangle\right\rangle \in\left(\widetilde{E}^{\Lambda}\right)^{\sim \Lambda^{\prime}}$ with $\tilde{\tilde{f}}(\alpha) \neq \tilde{\tilde{f}}(\beta)$. Put $h_{\lambda, \lambda^{\prime}}=f_{\lambda, \lambda^{\prime}}-f_{\lambda, \lambda^{\prime}}(x) \cdot u$, so $\left\langle\left\langle h_{\lambda, \lambda^{\prime}}\right\rangle\right\rangle \in\left(\widetilde{E}_{x}^{\Lambda}\right)^{\sim \Lambda^{\prime}}$. Then $\left\langle\left\langle h_{\lambda, \lambda^{\prime}}\right\rangle\right\rangle$ or $\left\langle\left\langle f_{\lambda, \lambda^{\prime}}(x)\right\rangle \cdot \tilde{g}\right\rangle$ separates $\alpha$ and $\beta$ for $\tilde{g} \in \widetilde{E}_{x}^{\Lambda}$ with $\tilde{g}(q) \neq 0$.

Theorem. Let $A$ be a uniformly closed subalgebra of $C_{0}(Y)$ for a locally compact Hausdorff space $Y$, and $I$ be a subalgebra of $A$ such that there are a finite number of subalgebras $I_{0}, I_{1}, \ldots, I_{n}$ of $A$ which satisfy the condition that $I_{k}$ is an ideal of $\mathrm{cl} I_{k-1}$ for every $k=1,2, \ldots, n$, where $I_{0}=A$ and $I_{n}=I$. Let $D$ be a plane domain containing the origin. Let $L$ be a linear subspace of finite dimension in $C_{0, R}(Y)$. Suppose that $\mathrm{Op}\left(I_{D}, \operatorname{Re} A+L\right)$ contains a function which is not harmonic on $D$. Then $I \mid K$ is uniformly closed and selfadjoint for every compact subset $K$ of $Y-\operatorname{Ker} I$ and $\operatorname{cl} I$ is selfadjoint.

Remark. If $I$ is an ideal of $A$, then the condition on $I$ and $A$ is satisfied with $n=1$. An ideal of an ideal need not be an ideal. Let $A(\bar{\Delta})$ be the disk 
algebra on the closed unit disk $\bar{\Delta}$. Let $I=\left\{f \in A(\bar{\Delta}): f(0)=f^{\prime}(0)=0\right\}$ and $J=\left\{f \in I: f^{\prime \prime \prime}(0)=0\right\}$. Then $I$ is an ideal of $A(\bar{\Delta})$ and $J$ is an ideal of $I$ while $J$ does not satisfy the condition $J \cdot A(\bar{\Delta}) \subset J$.

Proof of Theorem. The notations $\bar{Y}, \infty, \bar{Y}_{1}, p, \bar{Y}_{0}$, and $I^{\prime}$ are the same as in the proof of Theorem 2 in [5]. If $Y$ is not compact, then $\bar{Y}$ denotes the one point compactification of $Y$ and $\infty$ denotes the point in $\bar{Y}-Y$. If $Y$ is compact, then we add $\infty$ as an isolated point and $\bar{Y}$ denotes $Y \cup\{\infty\}$. We may suppose that $A$ is a closed subalgbera of $C(\bar{Y})$ such that $f(\infty)=0$ for every $f$ in $A$. Let $\bar{Y}_{1}$ be the quotient space obtained by identifying the points in $\bar{Y}$ which cannot be separated by $A$. Let $\bar{Y}_{0}$ be the quotient space obtained by identifying the points in $\bar{Y}$ which cannot be separated by $I$. Let $p$ be the point in $\bar{Y}_{0}$ which corresponds to the equivalence class in $\bar{Y}$ containing $\infty$. We may suppose that $\bar{Y}_{0}$ is the quotient space obtained by identifying points in $\bar{Y}_{1}$ which cannot be separated by $I$ and that $p$ corresponds to $\operatorname{Ker} I$. We may also suppose that each point in $\bar{Y}_{0}-\{p\}$ corresponds to a point in $\bar{Y}_{1}-\operatorname{Ker} I$, that is, we may suppose that $\bar{Y}_{0}-\{p\}=\bar{Y}_{1}-\operatorname{Ker} I$. Let $I^{\prime}=\operatorname{cl} I+C$ be the sum of the uniform closure of $I$ and the space of constant functions $C$. Then $I^{\prime}$ is a function algebra on $\bar{Y}_{0}$. Let $\operatorname{Ch}\left(I^{\prime}\right)$ be the Choquet boundary for $I^{\prime}$. We consider two cases. They are different from those of the proof of Theorem 2 in [5] : (1) There is no accumulation point of $\operatorname{Ch}\left(I^{\prime}\right)$ which is a point in $\mathrm{Ch}\left(I^{\prime}\right)$, or $p$ is the only accumulation point of $\mathrm{Ch}\left(I^{\prime}\right)$ which is a point in $\mathrm{Ch}\left(I^{\prime}\right)$. (2) There is an accumulation point of $\mathrm{Ch}\left(I^{\prime}\right)$ which is also a point in $\mathrm{Ch}\left(I^{\prime}\right)$ and is not $p$.

Case (1). Let $\Gamma$ be the Shilov boundary for $I^{\prime}$. We may suppose that $I^{\prime}$ is a function algebra on $\Gamma$. We show that $\{x\}$ is itself a compact neighborhood of $x$ for every $x$ in $\operatorname{Ch}\left(I^{\prime}\right)-\{p\}$. Since $x$ is not an accumulation point of $\mathrm{Ch}\left(I^{\prime}\right)$, there is an open neighborhood $U_{x}$ in $\Gamma$ of $x$ with $U_{x} \cap \mathrm{Ch}\left(I^{\prime}\right)=\{x\}$. If a point $y$ is in $U_{x}-\{x\}$, then $y$ is in $\Gamma$. So there is an open neighborhood $V_{y}$ in $\Gamma$ of $y$ such that $V_{y} \subset U_{x}$ and $x$ is not contained in $V_{y}$. Since $V_{y} \cap \mathrm{Ch}\left(I^{\prime}\right) \subset U_{x} \cap \mathrm{Ch}\left(I^{\prime}\right)=\{x\}$ and $x$ is not contained in $V_{y}$ we have $V_{y} \cap \mathrm{Ch}\left(I^{\prime}\right)=\varnothing$, which is a contradiction since $y \in \Gamma$ and $\operatorname{Ch}\left(I^{\prime}\right)$ is dense in $\Gamma$. We conclude that $U_{x}=\{x\}$, so $\{x\}$ is a compact neighborhood of $x$. We see by Lemma 1 that $I^{\prime} \mid \Gamma=C(\Gamma)$ since $I^{\prime} \mid U_{x}=C\left(U_{x}\right)$ for every $x$ in $\mathrm{Ch}\left(I^{\prime}\right)-\{p\}$. It follows that $\bar{Y}_{0}=\Gamma$ and $I^{\prime}=C\left(\bar{Y}_{0}\right)$. The rest of the proof is the same as in case (1) of the proof of Theorem 2 in [5].

Case (2). Choose a point $q$ in $\mathrm{Ch}\left(I^{\prime}\right)$ which is also an accumulation point of $\mathrm{Ch}\left(I^{\prime}\right)$ other than $p$. Suppose that $h$ is a function in $\mathrm{Op}\left(I_{D}, \operatorname{Re} A+L\right)$ which is not harmonic on $D$. First we show that $h$ is continuous on $D$. Suppose not. There is a point $a$ in $D$ such that $h$ is not continuous at $a$. There is a function $k$ in $I$ such that $k(q)=d>0 .\|k\|_{\infty} \leq 1$. By the proof of Lemma $1, \operatorname{Ch}\left(I^{\prime}\right)-\{p\}=\mathrm{Ch}(\operatorname{cl} I)$, so $q$ is a point in $\mathrm{Ch}(\operatorname{cl} I)$. Thus there is a function $u$ in $\operatorname{cl} I$ with $u(q)=1,\|u\|_{\infty} \leq 1$. Choose an analytic function $H$ defined on the open unit disk $\Delta$ with range in $D$, such that $H(0)=0$ and $a \in H(\Delta)$. Such a function exists since $D$ is connected. Let $r e^{i \theta}$ be a point in $\Delta$ with $H\left(r e^{i \theta}\right)=a$. So $H\left(r e^{i \theta} u\right)$ is a function in $\mathrm{cl} I$ such that $H\left(r e^{i \theta} u\right)(q)=a$ and $H\left(r e^{i \theta} u\right)\left(\bar{Y}_{0}\right)$ is a compact subset of $D$. In particular, there is a positive $\varepsilon$ with $\bigcup_{y \in \bar{Y}_{0}}\left\{z \in C:\left|z-H\left(r e^{i \theta} u\right)(y)\right| \leq 2 \varepsilon\right\} \subset D$. There 
is a function $\psi$ in $I$ with $\psi(q)=1$. Choose a function $\psi_{1}$ in $I$ with $\left\|\psi_{1}-H\left(r e^{i \theta} u\right)\right\|_{\infty\left(\bar{Y}_{0}\right)}<\varepsilon /\left(2\|\psi\|_{\infty\left(\bar{Y}_{0}\right)}\right)$. Put

$$
\psi_{q}=\psi_{1}-\left(\psi_{1}(q)-a\right) \psi,
$$

so $\psi_{q}(q)=a$. For a point $y$ in $\bar{Y}_{0}$, suppose that $z$ is a complex number with $\left|z-\psi_{q}(y)\right| \leq \varepsilon$. Then we have $\left|z-H\left(r e^{i \theta} u\right)(y)\right| \leq 2 \varepsilon$. We conclude that

$$
\bigcup_{y \in \bar{Y}_{0}}\left\{z \in C:\left|z-\psi_{q}(y)\right| \leq \varepsilon\right\} \subset D .
$$

As in the proof of Theorem 2 in [5] we can choose a sequence $\left\{q_{n}\right\}$ in $\mathrm{Ch}\left(I^{\prime}\right)$ with an accumulation point $q_{0}^{\prime \prime}$ and a function $f$ in $\operatorname{cl} I$ such that $h\left(f k+\psi_{q}\right) \in \operatorname{Re} A+L$ and that $h\left(f k+\psi_{q}\right)\left(q_{n}\right)$ does not converge to $h\left(f k+\psi_{q}\right)\left(q_{0}^{\prime \prime}\right)$, which is a contradiction. Thus we may assume that $h$ is continuous on $D$ in case (2). Choose a point $a$ in $D$ such that $h$ is not harmonic on any open neighborhood of $a$. We show that for every $x$ in $\operatorname{Ch}(\operatorname{cl} I)$ there exists a compact neighborhood $G_{x}$ of $x$ with $\mathrm{cl} I \mid G_{x}=C\left(G_{x}\right)$. If so, $\operatorname{cl} I$ is selfadjoint by Lemma 1 . Let $x$ be a point in $\mathrm{Ch}(\operatorname{cl} I)$. Then there is a function $\phi$ in $I$ with $\phi(x)=a$ and $\phi\left(\bar{Y}_{0}\right) \subset D$ in the same way as above. Let $f_{1}$ be a function in $I$ with $f_{1}(x)=1$. Put $f_{0}=f_{1}^{n+1}$. Since cl $I_{k}$ is an ideal of $\operatorname{cl} I_{k-1}$ by the condition on $I_{k}$ we have $f_{1}^{k} \cdot A \subset \operatorname{cl} I_{k}$. Thus $f_{0} \cdot A \subset I$ and $f_{0} \cdot A$ is a Banach algebra contained in $C\left(\bar{Y}_{0}\right)$ with respect to the norm defined by

$$
\|u\|_{f_{0} \cdot A}=\inf \left\{\left\|f_{0}\right\|_{\infty} \cdot\|g\|_{\infty}: g \in A, u=f_{0} g\right\}
$$

for $u$ in $f_{0} \cdot A$. Without loss of generality we may assume $(\operatorname{Re} A) \cap L=$ $\{0\} . \operatorname{Re} A+L$ is a Banach space with respect to the norm defined by

$$
\|u+v\|_{\operatorname{Re} A+L}=\|u\|_{\operatorname{Re} A}+\|v\|_{\infty}
$$

for every $u \in \operatorname{Re} A$ and $v \in L$ by Lemma 3 . We show that $f_{0} \cdot A$ is ultraseparating near $x$. Let $\varepsilon$ be a positive number such that $d\left(\phi\left(\bar{Y}_{0}\right), D^{c}\right)>\varepsilon$, where $d(\cdot, \cdot)$ is the usual Euclidian distance and $D^{c}$ is the complement of $D$ in the complex plane $C$. Since $h$ is not harmonic near $a$, we see that

$$
\left|\Delta_{1}\left(h_{\eta}\left(z, z^{\prime}\right)\right)\right| \geq(1 / 2)\left|\Delta_{1}\left(h_{\eta}(a, 1)\right)\right| \neq 0
$$

on $\left\{\left(z, z^{\prime}\right) \in C^{2}:|z-a|<\varepsilon^{\prime \prime},\left|z^{\prime}-1\right|<\varepsilon^{\prime \prime}\right\}$ for a suitably chosen $\eta$ with $\varepsilon /\left(2\left\|f_{0}\right\|_{\infty}\right)>\eta>0$ and a suitably chosen smoothing operator $\sigma_{\eta}$ and an $\varepsilon>\varepsilon^{\prime \prime}>0$, where

$$
h_{\eta}\left(z, z^{\prime}\right)=\iint h\left(z-z^{\prime} w\right) \sigma_{\eta}(w) d x d y
$$

in the same way as in $[4, \mathrm{pp} .634,635]$. Since $L$ is finite dimensional, we have $\operatorname{cl}(\operatorname{Re} A+L)=(\operatorname{cl} \operatorname{Re} A)+L$. So we see that

$$
h_{\eta}\left(\phi+g_{1} f_{0}^{2} t, f_{0}\right) \in(\operatorname{cl} \operatorname{Re} A)+L
$$

for $g_{1} \in A+C$ and a complex number $t$ with sufficiently small absolute value. Thus by Lemma 5 in [4]

$$
\left|g_{1}\right|^{2} \mid \Delta_{1}\left(h_{\eta}\left(\phi, f_{0}\right) \cdot\left|f_{0}\right|^{4}\right) \in(\operatorname{cl} \operatorname{Re} A)+L .
$$


So by the Stone-Weierstrass theorem we see that

$$
C\left(\bar{Y}_{1}\right) \cdot \Delta_{1}\left(h_{\eta}\left(\phi, f_{0}\right) \cdot\left|f_{0}\right|^{4}\right) \subset(\operatorname{cl} \operatorname{Re} A)+L .
$$

Let $G_{x}$ be a compact neighborhood of $x$ such that

$$
G_{x}=\left\{y \in \bar{Y}_{0}:|\phi(y)-a| \leq \varepsilon^{\prime \prime} / 2,\left|f_{0}(y)-1\right| \leq \varepsilon^{\prime \prime} / 2 \mid\right\},
$$

so $\Delta_{1}\left(h_{\eta}\left(\phi, f_{0}\right) \cdot\left|f_{0}\right|^{4}\right)$ never take zero on $G_{x}$. Thus we have

$$
C\left(G_{x}\right)=(\operatorname{cl} \operatorname{Re} A+L) \mid G_{x} .
$$

Let $\Lambda$ be a discrete space with cardinality not less than that of an open base for the topology of $Y$ at $x$. By Lemma 3 we see that $C\left(F_{x}^{\Lambda}\right)=(\operatorname{cl} \operatorname{Re} A)^{\sim \Lambda} \mid G_{x}$. Thus for different points $p$ and $q$ in $F_{x}^{\Lambda}$ there is a function $\left\langle u_{\lambda}\right\rangle$ in $(\operatorname{cl} \operatorname{Re} A)^{\sim \Lambda}$ with $\left\langle u_{\lambda}\right\rangle(p) \neq\left\langle u_{\lambda}\right\rangle(q)$, in particular, we may suppose that $u_{\lambda} \in \operatorname{Re} A$. Then there exists $\nu_{\lambda} \in \operatorname{Re} A$ for every $\lambda$ such that $u_{\lambda}+i \nu_{\lambda} \in A$, so $\exp \left(u_{\lambda}+i \nu_{\lambda}\right) \in A$ and since $\left\|\exp \left(u_{\lambda}+i \nu_{\lambda}\right)\right\|_{\infty\left(\bar{Y}_{1}\right)}=\left\|\exp u_{\lambda}\right\|_{\infty\left(\bar{Y}_{1}\right)}$ we see that $\left\langle\exp \left(u_{\lambda}+i \nu_{\lambda}\right)\right\rangle \in \widetilde{A}^{\Lambda}$. By Lemma 4 in [5] we see

$$
\left\langle f_{0} \cdot \exp \left(u_{\lambda}+i \nu_{\lambda}\right)\right\rangle=\left\langle f_{0}\right\rangle \cdot\left\langle\exp \left(u_{\lambda}+i \nu_{\lambda}\right)\right\rangle=\left\langle\exp \left(u_{\lambda}+i \nu_{\lambda}\right)\right\rangle
$$

on $F_{x}^{\Lambda}$. By the definition of $\|\cdot\|_{f_{0}} \cdot A$ we have

$$
\left\langle f_{0} \cdot \exp \left(u_{\lambda}+i \nu_{\lambda}\right)\right\rangle \in\left(f_{0} \cdot A\right)^{\sim \Lambda}
$$

and it separates $p$ and $q$. So by Theorem $1(1)$ of [5], $f_{0} \cdot A$ is ultraseparating near $x$. Put $B=\left\{g \in f_{0} \cdot A: g(x)=0,\|g\|_{f_{0} \cdot A} \leq \varepsilon\right\}$. In the same way as in the proof of Lemma 1.2 in [4] (cf. [5, Lemma 11]) we see the following.

There are positive integers $n_{0}$ and a real number $\varepsilon^{\prime}$ with $0<\varepsilon^{\prime}<\varepsilon$, and a function $g_{0}$ in $B$ such that

$$
\left\{g \in f_{0} \cdot A: g(x)=0,\left\|g-g_{0}\right\|_{f_{0} \cdot A}<\varepsilon^{\prime}\right\} \subset B,
$$

and there is a dense (with respect to the topology induced by the norm $\|\cdot\|_{f_{0} \cdot A}$ ) subset $U$ in $\left\{g \in f_{0} \cdot A: g(x)=0,\left\|g-g_{0}\right\|_{f_{0} \cdot A}<\varepsilon^{\prime}\right\}$ which satisfies that for every $g$ in $U$ we have

$$
h(g+\phi) \in \operatorname{Re} A+L \text { and }\|h(g+\phi)\|_{\operatorname{Re} A+L}<n_{0} .
$$

Let $U_{0}=\left\{g \in f_{0} \cdot A: g+g_{0} \in U\right\}$. Then $U_{0}$ is dense (with respect to the topology induced by $\left.\|\cdot\|_{f_{0} \cdot A}\right)$ in $\left\{g \in f_{0} \cdot A: g(x)=0,\|g\|_{f_{0} \cdot A} \leq \varepsilon^{\prime}\right\}$. Put $F=$ $F_{x}^{\Lambda}, S=\left(f_{0} \cdot A_{x}\right)^{\sim \Lambda}\left|F_{x}^{\Lambda}, S_{0}=\widetilde{U}_{0}^{\Lambda}\right| F_{x}^{\Lambda}, Q=[\{x\} \times \Lambda], h_{0}(z)=h(z+a), \delta=\varepsilon^{\prime}$, and $T=\operatorname{Re} \widetilde{A}^{\Lambda} \mid F_{x}^{\Lambda}$. Then the conditions of Lemma 2 are satisfied. We check this. $F$ is trivially a compact Hausdorff space and $Q$ is a compact subset of $F$, and $S$ is a Banach algebra contained in $\{\tilde{f} \in C(F): \tilde{f} \mid Q=0\}$. We see that the separation and ultraseparation conditions on $S$ are satisfied by Lemma 4. We see that $Q=\{p \in F: \tilde{f}(p)=0$ for every $\tilde{f}$ in $S\}$ by Proposition 2 in [5]. Let $\left\langle g_{\lambda}\right\rangle \in \widetilde{U}_{0}^{\Lambda}$. Then $h\left(\left\langle g_{\lambda}\right\rangle+\left\langle g_{0}\right\rangle+\langle\phi\rangle\right)$ is in $(\operatorname{Re} A+L)^{\sim \Lambda}$ by the definition of $U_{0}$. Thus we see that $h(\tilde{g}+a)$ is in $\operatorname{Re} \tilde{A}^{\Lambda} \mid F_{x}^{\Lambda}$ for every $\tilde{g}$ in $\tilde{U}_{0}^{\Lambda} \mid F_{x}^{\Lambda}$ by Lemma 4 in [5] and Lemma 3. Equivalently $h_{0}(\tilde{g}) \in T$ for every $\tilde{g} \in S_{0}$. Thus by Lemma 2 we see that for every point $p$ in $F_{x}^{\Lambda}-[\{x\} \times \Lambda]$ there is a compact neighborhood $O_{p}$ of $p$ in $F_{x}^{\Lambda}$ with $O_{p} \subset F_{x}^{\Lambda}-[\{x\} \times \Lambda]$ such that

$$
\left\{\tilde{u} \in \operatorname{Re} \widetilde{A}^{\Lambda}\left|F_{x}^{\Lambda}: \tilde{u}\right|[\{x\} \times \Lambda]=0\right\} \mid O_{p}=C_{R}\left(O_{p}\right) .
$$


We will see that

$$
\left\{\tilde{u} \in \operatorname{Re} \widetilde{A}^{\Lambda}\left|F_{x}^{\Lambda}: \tilde{u}\right|[\{x\} \times \Lambda]=0\right\}=\operatorname{Re} \widetilde{A}_{x}^{\Lambda} \mid F_{x}^{\Lambda} .
$$

$\left\{\tilde{u} \in \operatorname{Re} \widetilde{A}^{\Lambda}\left|F_{x}^{\Lambda}: \tilde{u}\right|[\{x\} \times \Lambda]=0\right\} \supset \operatorname{Re} \widetilde{A}_{x}^{\Lambda} \mid F_{x}^{\Lambda}$ is trivial. We show the inverse inclusion. If $\tilde{u} \in \operatorname{Re} \tilde{A}^{\Lambda} \mid F_{x}^{\Lambda}$ with $\tilde{u} \mid[\{x\} \times \Lambda]=0$, then there is $\left\langle u_{\lambda}+i \nu_{\lambda}\right\rangle$ in $\widetilde{A}^{\Lambda}$ such that $\left\langle u_{\lambda}\right\rangle \mid F_{x}^{\Lambda}=\tilde{u}$. Put $\left\langle u_{\lambda}+i \nu_{\lambda}\right\rangle-\left\langle i \nu_{\lambda}(x) \cdot f_{0}\right\rangle \in \tilde{A}^{\Lambda}$. Since $u_{\lambda}+i \nu_{\lambda}-i \nu_{\lambda}(x) \cdot f_{0}$ is in $A_{x}$, it follows that $\left\langle u_{\lambda}+i \nu_{\lambda}\right\rangle-\left\langle i \nu_{\lambda}(x) \cdot f_{0}\right\rangle \in \widetilde{A}_{x}^{\Lambda}$ and $\operatorname{Re}\left(\left\langle u_{\lambda}+i \nu_{\lambda}\right\rangle-\left\langle i \nu_{\lambda}(x) \cdot f_{0}\right\rangle\right) \mid F_{x}^{\Lambda}=\tilde{u}$ by Lemma 4 in [5]. Thus we have

$$
\left(\operatorname{Re} \widetilde{A}_{x}^{\Lambda}\right) \mid O_{p}=C_{R}\left(O_{p}\right)
$$

We may suppose that $\operatorname{cl}\left(\widetilde{A}_{x}^{\Lambda} \mid F_{x}^{\Lambda}\right)+C$ is a function algebra on ${ }_{0} F_{x}^{\Lambda} \quad\left({ }_{0} F_{x}^{\Lambda}\right.$ is the quotient space of $F_{x}^{\Lambda}$ obtained by identifying the points in $[\{x\} \times \Lambda]$ ) by Proposition 2 in [5] since $A$ is ultraseparating near $x$. By a theorem of Hoffman and Wermer, and Bernard $[1,6]$ on the uniformly closed real part of a Banach function algebra we see that

$$
\left(\operatorname{cl}\left(\widetilde{A}_{x}^{\Lambda} \mid F_{x}^{\Lambda}\right)+C\right) \mid O_{p}=C\left(O_{p}\right),
$$

so by Corollary 2.13 in [2] we see that

$$
\operatorname{cl}\left(\tilde{A}_{x}^{\Lambda} \mid F_{x}^{\Lambda}\right)+C=C\left({ }_{0} F_{x}^{\Lambda}\right)
$$

It follows that

$$
\operatorname{cl}\left(\widetilde{A}^{\Lambda} \mid F_{x}^{\Lambda}\right)=C\left(F_{x}^{\Lambda}\right) .
$$

We see that $A \mid G=C(G)$ for a compact neighborhood $G$ of $x$ by Theorem 1 (2) in [5]. It follows that $I \mid G^{\prime}=C\left(G^{\prime}\right)$ for a compact neighborhood $G^{\prime}$ of $x$ in $\bar{Y}_{0}-\{p\}$. The results follow.

As in Corollary 1.1 in [4] we see the following.

Corollary. Let $A$ and $I$ and $L$ be the same as in the theorem. Let $S$ be an interval of the real line. Suppose that one of the following holds.

(1) $\mathrm{Op}\left(I_{D}, A\right)$ contains a nonanalytic function on $D$.

(2) $\mathrm{Op}\left((\operatorname{Re} I)_{S}, \operatorname{Re} A+L\right)$ contains a nonaffine function on $S$.

Then $I \mid K$ is uniformly closed and selfadjoint for every compact subset $K$ of $Y-\operatorname{Ker} I$ and $\operatorname{cl} I$ is selfadjoint.

\section{REFERENCES}

1. A. Bernard, Espace des parties réeles des éléments d'une algèbre de Banach de fonctions, $\mathrm{J}$. Funct Anal. 10 (1972), 387-409.

2. R. B. Burckel, Characterizations of $C(X)$ among its subalgebras, Marcel Dekker, New York, 1972.

3. O. Hatori, Functions which operate on the real part of a function algebra, Proc. Amer. Math. Soc. 83 (1981), 565-568.

4. _ Range transformations on a Banach function algebra, Trans. Amer. Math. Soc. 297 (1986), 629-643.

5. __ Range transformations on a Banach funtion algebra. II, Pacific J. Math. 138 (1989), 89-118.

6. K. Hoffman and J. Wermer, A characterization of $C(X)$, Pacific J. Math. 12 (1962), 941-944.

Tokyo Medical College, Department of Mathematics, 6-1-1 Shinjuku, Shinjuku-ku, TOKYO, 160 JAPAN

E-mail address: BEROBERO@JPNWAS00.bitnet 